\title{
Lorentz Transform in Multi-Dimensional Space
}

\author{
I. A. Urusovskii \\ Acoustics N. N. Andreev Institute, Moscow, Russia \\ Email: urusovskii_ia@mail.ru
}

Received August 23, 2012; revised September 30, 2012; accepted October 9, 2012

\begin{abstract}
It is shown that in Euclidean space with any number of spatial dimensions more than three, the Lorentz transform holds true if the proper time of each elementary particle is proportional to the length of its path in the extra-dimensional subspace, and all elementary particles move at the speed of light in the complete space. The six-dimensional treatment of the Coulomb force of interaction between two charges is given. The electric force is due to the motion of charges in the extra-dimensional subspace and is equal to the corresponding Lorentz force.
\end{abstract}

Keywords: Lorentz Transform; Euclidean Multi-Dimensional Space; Compton Wave Length; De Broglie Waves; Spin and Isotopic Spin; Heisenberg Uncertainty; CPT Symmetry; Coulomb Force

\section{Introduction}

It is commonly assumed that space-time is pseudoEuclidian while the interval $d s$ of relativity theory is an element of the trajectory of a particle in this space-time. But for any Euclidean space having more than three spatial dimensions, the Lorentz transform may be obtained if the elementary particle is moving at the speed of light in the complete multi-dimensional space and the proper time of this particle is proportional to its path length in extra-dimensional subspace $(Y)$ of the whole space. This explains the twins paradox as well: the more rapidly a body moves in three-dimensional subspace $(X)$, the lower its velocity in extra-dimensional subspace and, conesquently, the slower its proper time passes. This assumption goes back to F. Klein's idea [1,2] of the motion of particles with the speed of light in multi-dimensional space where mechanics is represented as quasi-optics.

The light and also particles of substance have corpuscular as well as wave properties, examples of which are the diffraction of electrons when they are represented as a wave and photoelectric emission when photons are represented as particles. These properties testify that several basic properties of light and particles are the same. The main property of light is that it propagates, in the absence of gravitation, at the speed of light in any reference frame. Then elementary particles of substance must also move at the same speed. This is impossible in three-dimensional space but possible in multi-dimensional space.

All directions in extra-dimensional subspace $Y$ of the multi-dimensional Euclidean space considered are perpendicular to any direction in three-dimensional sub- space $X$. Therefore projections $d s$ and $d x$ of a displacement $c d t$ of a particle, moving at the speed of light in the complete Euclidean space of any number of spatial dimensions more than three, on subspace $X$ and subspace $Y$, respectively, are connected by the Pythagorean theorem: $(c d t)^{2}=(d s)^{2}+(d x)^{2}$, where $c d t$ is a displacement of the particle par time lapse $c d t$. From this, one obtains the metric

$$
(d s)^{2}=(c d t)^{2}-(d x)^{2} .
$$

Particles should be confined to a small vicinity of the three-dimensional Universe by the forces (of cosmological nature) orthogonal to it. In the Euclidean space under consideration, elementary particles cannot be well off the subspace $X$ at large Compton distances and must be confined near this subspace. Otherwise, there would be neither compact trajectories of elementary particles in extra-dimensional subspace nor macroscopic bodies. Such forces (of the Lorentz type) are perpendicular to any direction in three-dimensional subspace and are the reason why the trajectories of elementary particles are compacted in extra-dimensional subspace despite the centripetal forces in it. Thus, projections of these trajectories on additional subspace are finite ones, and elementary particles move at the speed of light, remaining at rest, on average, in this subspace. For this reason atoms and macroscopic bodies consisting of elementary particles may be at rest as a whole.

The dispersion equation is the same for the acoustic waveguide, the electromagnetic one, and de Broglie waves: $v_{p h} v_{g}=c^{2}$, where $v_{p h}$ is the phase velocity, $v_{g}$ the group velocity, $c$ the speed of waves in a free 
medium (the speed of sound in the first case and that of light in the other two cases). The main characteristic of any waveguide is that it has finite transverse dimensions. The dispersion of waves is due to these dimensions only. This indicates that the part of space which we deal with in the experiment is only approximately three-dimensional and has rather small (Compton) sizes in the additional subspace.

Energy propagates along rays. That is why the speed of its propagation along the waveguide is less than the speed of light and is equal to $c \sin \theta$, the group velocity, where $\theta$ is the angle of inclination of the trajectory of an elementary particle to the transverse section of the waveguide. Wave fronts are perpendicular to the trajectory. Therefore, these fronts move along the waveguide more rapidly than the speed of light and their phase velocity is $c / \sin \theta$, the speed of an imagined tachyon, so that the product of the group and phase velocities is $c^{2}$. The dispersion is formed by the walls of the waveguide, if it has them, or force fields, as for an electron moving in a magnetic field along a helical line; this is also a waveguide of a sort. Here the dispersion equation is due to the perpendicularity of trajectories to the wave fronts.

The energy of a photon is equal to $h v$, where $v$ is the frequency of light and $h$ the Plank constant. By virtue of a principle of similarity of the basic properties of substance and light concretizing the principle of simplicity [3], the rest energy $m c^{2}$ of a particle may be represented as a quantum of energy $h v$, so that

$$
m c^{2}=h v
$$

The unique and natural frequency $v$ for a particle of substance at rest in $X$ is the frequency of its rotations in extra-dimensional subspace $Y$. On the other hand, the particle moves with the speed of light along the directrix of the motion tube, from which $2 \pi a=c / v$, where $a$ is the radius of the tube. Eliminating $v$ from this equality and (2), one finds $2 \pi a=h / m c, a=\hbar / m c$; that is, the length of the directrix is equal to the Compton wavelength. The length of the directrix corresponds to the period $h$ of the coordinate of action in 5-optics [4].

There is no need to consider the complete space as a pseudo-Euclidean one. Let us demonstrate that the Lorentz transform may by obtained for the Euclidean space under consideration.

The particle moves along the cylinder surface (the motion tube as the envelope of the geodesics) with Compton radius $a=\hbar /(m c)$ and with its axis in the subspace $X$ and directrix in the subspace $Y$. The interval $d s$ is a projection of the displacement of a particle on the directrix of the motion tube, and $d x$ is a projection of the displacement on the tube axis (see Figures 1 and 2).

The particle which is stationary in the projection onto
$X$ in the inertial frame of reference $K$ moves at the speed of light $c$ and travels, in the simplest case, in a circle within the three-dimensional subspace $Y$ that complements $X$ with respect to the complete Euclidean space $R_{6}$ while the centre of the circle is in $X$. In any other inertial frame of reference this particle moves along a helical line (curve 1 in Figure 2) located on the cylindrical surface (motion tube) in $R_{6}$ with its axis belonging to $X$.

Let us assume that the proper time of the particle is proportional to the length of its path in $Y$ because it is a natural measure of time. This length is proportional to $|\cos \theta|$, where $\theta$ is the angle of inclination of the helix with respect to the tube directrix. If the particle makes one rotation within the proper time $\tau$, for a fixed observer with respect to whom the particle moves along the tube with the velocity $v=c \sin \theta$, this will occur within the time $v=c \sin \theta$, where

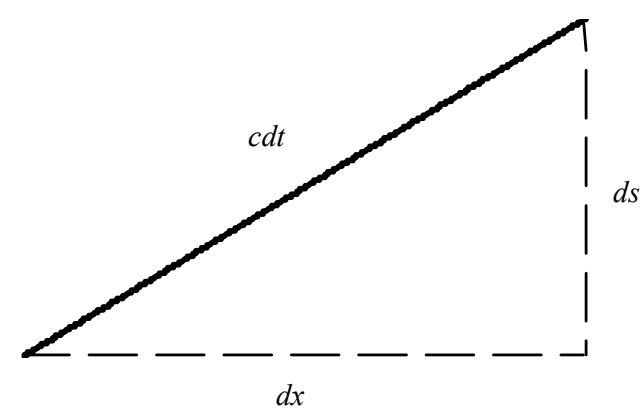

Figure 1. Projections $d s$ and $d x$ of a displacement $c d t$ of a particle moving at the speed of light in the complete multi-dimensional Euclidean space.

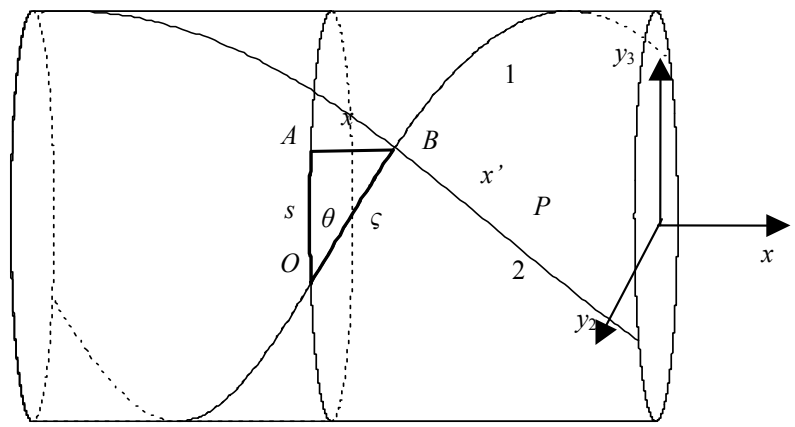

Figure 2. 1 is the helical trajectory of a particle moving in six-dimensional space with the speed of light $c$ along the cylinder surface (the envelope of the geodesics) of Compton radius $a=\hbar /(m c)$ with its axis in the subspace $X$ and directrix in the subspace $Y . \varsigma=c t .2$ is a helical line of equal proper time of this particle. It passes through the particle perpendicularly to the helical trajectory. It moves along the same cylinder surface with the velocity of the de Broglie wave. Its pitch is equal to the de Broglie wavelength. In subspace $X$ the particle moves at the speed $v=c \sin \theta$, where $\theta$ is the angle of inclination of the trajectory of an elementary particle to the transverse section of that cylinder. 


$$
\sin \theta=v / c, \cos \theta= \pm \sqrt{1-(v / c)^{2}}
$$

In (3) and the subsequent discussion the plus sign applies to the particle rotating about the axis of the tube in the positive direction and the minus sign applies to the antiparticle rotating in the opposite direction. Intervals of the proper time of the particle (or antiparticle) $d \tau$ and of the fixed observer's time $d t$ are related by the formula

$$
d t= \pm d \tau / \cos \theta=d \tau / \sqrt{1-(v / c)^{2}}
$$

In the fixed frame of reference $K$ the particle has a component of the velocity $c \cos \theta$ along the directrix. For the fixed observer, the proper time of the particle is, according to (4), also proportional to $\cos \theta$ so that the particle moves with the velocity $c$ in its proper frame $\left(K^{\prime}\right)$ of reference as well.

A particle at rest in $K$, a particle moving with the speed of light $c$ along the directrix, displaces per proper time $d \tau$ in an interval $d s$ equal to

$$
d s= \pm c d \tau \text {. }
$$

The momentum of this particle is a vector directed along the tangent to the directrix at a point where this particle is placed at a given time. The magnitude of this vector is $m c$, which is the product of the mass $m$ of the particle and its speed $c$. This is the momentum at rest in relativistic mechanics. The energy at rest $E_{0}$, according to this definition, is the product of momentum and the speed of a particle: $E_{0}=m c^{2}$. In the general case, the total momentum of a particle is the vector directed along the tangent to the helical trajectory. Its value $p$ is the product of the mass $m$ of a particle and the ratio of its path

$$
d \varsigma=c d t
$$

in the whole space to the proper time $d \tau$ expended for this path:

$$
p=m \frac{d \varsigma}{d \tau}=\frac{m c}{|\cos \theta|}=m c / \sqrt{1-(v / c)^{2}} .
$$

This is the relativistic formula for the total momentum of a particle. Projections $p x$ and $p y$ of the total momentum on the generatrix and directrix of a tube are equal to the spatial and temporal components of the four-momentum of a particle, respectively $[5,6]$ :

$$
p_{x}= \pm m c \tan \theta=m v / \sqrt{1-(v / c)^{2}}, p_{y}= \pm m c .
$$

In the general case, $\theta \neq 0$ and the total energy of a particle $E$ is the product of the total momentum $p$ and the speed of movement $c$ along a helix:

$$
E=p c=\frac{m c^{2}}{|\cos \theta|}=m c^{2} / \sqrt{1-(v / c)^{2}} \text {. }
$$

This value is the total relativistic energy of a particle. Note that the ratio of the total energy to the total momentum of the particle is the same as that for a photon. This is yet another common property of light and substance.

Displacement of the particle over the interval $d s$ along the motion tube directrix and the corresponding rotation through the angle $d \alpha=d s / a$ about the axis of the tube, where $a$ is the radius of the tube, are identical in any frame of reference. Designating the projection of particle displacement $d \varsigma$ over the surface of the tube onto its generatrix by $d x$ in the frame of reference $K$ and using the Pythagorean theorem for the rectangular triangle $O A B$ shown in Figure 2, one obtains (1). If this relation is considered as the initial one, then (6) follows from it; that is, the particle moves in $R_{6}$ at the speed $c$.

The projection of the sides of the triangle $O A B$ on the trajectory of the particle gives

$$
s \cos \theta+x \sin \theta=\varsigma .
$$

Let us write the initial conditions in the form $t=\tau=0$ with $x=s=0$. Then, referring to (4) and (5), it follows that:

$$
s= \pm c \tau, \varsigma=c t .
$$

Substituting $\sin \theta=v / c \quad$ (2) and (10) into (9) gives the Lorentz transform for time:

$$
\tau= \pm[t-(x / c) \sin \theta] / \cos \theta=\left[t-\left(x v / c^{2}\right)\right] / \sqrt{1-(v / c)^{2}} .
$$

A similar consideration applied to the system of reference $K^{\prime}$, taking into account that the system $K$ moves relative to the considered particle with velocity $-v$, leads to the reversed transform:

$$
\begin{aligned}
& t= \pm\left[\tau+\left(x^{\prime} / c\right) \sin \theta\right] / \cos \theta \\
& =\left[\tau+\left(x^{\prime} v / c^{2}\right)\right] / \sqrt{1-(v / c)^{2}} .
\end{aligned}
$$

where $x^{\prime}$ is the coordinate along the line of equal proper time. The transition from the system $K$ to $K^{\prime}$ corresponds to a turn through an angle $-\theta$ about the origin $x=s=0$ of coordinate net $x, s$ on the surface of the motion tube, together with trajectories of particles on it. This turn transfers a helical trajectory in the directrix of the tube.

For a geometrical interpretation of the rest Lorentz transform, let us consider a trajectory of a particle moving along the tube with the same velocity $v$ and intersecting at a time $t=0$ with the helix $s \cos \theta+x \sin \theta=0$ at an arbitrary point $P$. In the system of reference $K$, trajectories inclined at the angle $\theta$ to the directrix are the lines of the constant coordinate $x^{\prime}$ of the system $K^{\prime}$. The coordinate $x^{\prime}=B P$ is measured along the helix described by Equation (9). The measurement is taken from the normal section of the tube $x=v t=\varsigma \sin \theta$ to 
the section reached by the particle $P$ at time $t$ when $x$ is the distance between $P$ and the directrix $O A$. Projecting segments $x^{\prime}, x, \varsigma$, and $s$ (see Figure 3 as the resolvent of the cylinder in Figure 2) on the generatrix and directrix, the trajectory of the particle and the helix (along $x^{\prime}$ ) perpendicular to the trajectory are obtained with $\cos \theta>0$ :

$$
\begin{aligned}
& x^{\prime} \cos \theta+\varsigma \sin \theta=x, s \cos \theta+x \sin \theta=\varsigma, \\
& \varsigma \cos \theta-x^{\prime} \sin \theta=s, x \cos \theta-s \sin \theta=x^{\prime} .
\end{aligned}
$$

Dividing these equalities through by $\cos \theta$ and eliminating $s, \varsigma$, and $\theta$ by means of (3) and (10), according to which $s=c \tau, \varsigma=c t, \sin \theta=v / c$, and $\cos \theta=\sqrt{1-(v / c)^{2}}$ in the considered case, one may easily obtain the Lorentz transform in the standard form:

$$
\begin{aligned}
& x^{\prime}=(x-v t) / \sqrt{1-(v / c)^{2}}, \\
& \tau=\left[t-\left(x v / c^{2}\right)\right] / \sqrt{1-(v / c)^{2}}, \\
& t=\left[\tau+\left(x^{\prime} v / c^{2}\right)\right] / \sqrt{1-(v / c)^{2}}, \\
& x=\left(x^{\prime}+v t\right) / \sqrt{1-(v / c)^{2}} .
\end{aligned}
$$

The proper length of a moving rigid scale is the difference between the coordinates $x^{\prime}$ of its ends. In the system $K$, this is equal to the length of a segment of the helix perpendicular to the trajectories of particles moving with this segment between normal sections of the motion tube corresponding to those ends. It is a segment of the line of equal time in the system $K^{\prime}$. The length of the same scale in the system at rest $K$ is the difference between the coordinates $x$ of its ends. This is equal to thedistance along the generatrix between those normal

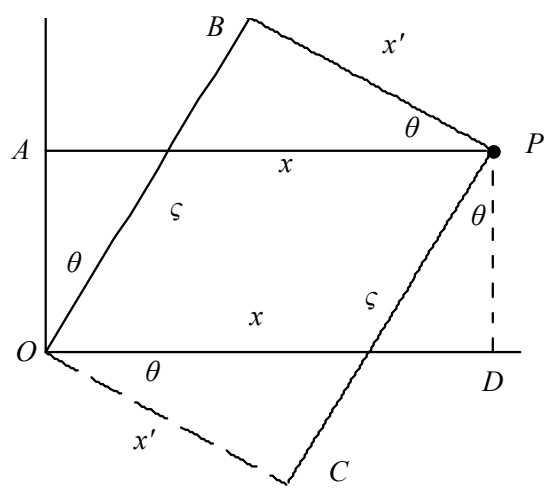

Figure 3. The evolvent of the motion tube shown in Figure 2; $s$ and $x$ are coordinates of a particle along the directrix of the motion tube and the tube axis, respectively, in the system $K$ at rest; $x^{\prime}$ is the same but along the generatrix in the system $K^{\prime}$ connected with the particle. $\varsigma=c t$,

$O A=P D=s, A P=O D=x, B P=O C=x^{\prime}$, $\boldsymbol{C P}=\boldsymbol{O B}=\varsigma$. sections, which is $1 / \cos \theta$ less than the proper length.

Thus, the Lorentz contraction of moving scales is a result of the projection of lengths in multi-dimensional space on three-dimensional space. Non-simultaneity of spatially spaced events in one system of reference with simultaneity in another is explained by non-parallelism of helixes of equal time in systems of reference moving relative to one another.

The above interpretation of Formula (4) also holds for the curved axis of a motion tube because in any case all normal sections of such a tube are perpendicular to any directions in the subspace $X$ to which the axis of the tube belongs.

Another helix placed on the same tube perpendicularly to the helical trajectory of a particle and passing through the particle is the line of equal proper time of the system $K^{\prime}$. This helix moves along the tube with the velocity of the de Broglie wave, $v_{p h}=c / \sin \theta=c^{2} / v$, where $v$ is the velocity of the particle in the subspace $X$. The pitch $\ell$ of this helix is equal to the de Broglie wavelength,

$\ell=2 \pi \frac{a}{|\tan \theta|}=\frac{h}{m c|\tan \theta|}=h / p_{x}=\frac{h}{|m v|} \sqrt{1-(v / c)^{2}}$, as is seen from (7) and Figures 2 and 3. The angle coordinate $s / a$ of the helix described by (9) and (10) is equal to $\frac{s}{a}=\frac{\varsigma}{a \cos \theta}-\frac{x}{a} \tan \theta=\frac{c t-x \sin \theta}{\cos \theta} \frac{m c}{\hbar}, \hbar=h / 2 \pi$, From this and from (6) and (7) it is seen that $s / a$ is equal to the phase of the de Broglie wave $\pm\left[E t-p_{x} x\right] / \hbar$. In the place of the position of the particle $x=v t$ this phase is an angle of rotation of the particle on the motion tube. The function $\Psi=\exp (i s / a)$ satisfies the Klein-Gordon equation

$$
a^{2} \frac{\partial^{2} \Psi}{\partial \varsigma^{2}}=a^{2} \frac{\partial^{2} \Psi}{\partial x^{2}}-\Psi
$$

The Heisenberg uncertainty relations are due to uncertainty of coordinates and moments of a particle in $Y$. In fact, let the directrix of a motion tube of a particle be displaced in the plane $y_{2}, y_{3}$. Then projections of the momentum of a particle on axes $y_{2}$ and $y_{3}$ and coordinates of the particle along these axes are equal to

$$
\begin{aligned}
& p_{y 2}=-m c \sin \varphi, p_{y 3}=m c \cos \varphi, \\
& y_{2}=\frac{\hbar}{m c} \cos \varphi, y_{3}=\frac{\hbar}{m c} \sin \varphi,
\end{aligned}
$$

where $\varphi$ is the angle of rotation of the particle about the axis of a tube reckoned from the axis $y_{2}$. The average over $\varphi$ values of coordinates and projections of the momentum are equal to zero but their mean-square values are equal to

$$
\left\langle y_{2}^{2}\right\rangle=\left\langle y_{3}^{2}\right\rangle=\frac{1}{2}\left(\frac{\hbar}{m c}\right)^{2},\left\langle p_{y 2}^{2}\right\rangle=\left\langle p_{y 3}^{2}\right\rangle=\frac{1}{2}(m c)^{2},
$$


from which the following relations can be found:

$\left\langle p_{y 2}^{2}\right\rangle \cdot\left\langle y_{2}^{2}\right\rangle=\left\langle p_{y 3}^{2}\right\rangle \cdot\left\langle y_{3}^{2}\right\rangle=\hbar^{2} / 4$.

The simple geometrical treatment of spin and isotopic spin needs three additional spatial dimensions. In sixdimensional Euclidean space, spin and isotopic spin are treated as projections of the total momentum on the subspace $X$ and subspace $Y$, respectively, while the proper magnetic moment is a result from rotation of the charge at the speed of light in $Y$ along the orbit of the Compton radius [5-7]. The first substantiation of space six-dimensionality was given by di Bartini [8], who calculated the fundamental physical constants. A four-dimensional treatment of Metric (1) with a somewhat different account of wave properties of space (as applied to four spatial dimensions) is given by Gribov [9].

The proper moment of momentum $S$ of a particle is a vector product of the proper momentum and radius vector of this particle. The component of the radius vector and the component of velocity of the particle on the axis of the motion tube are perpendicular to the plane of rotation in $Y$ and therefore do not make any contribution to $\boldsymbol{S}$. Hence, for a particle moving in six-dimensional space along a helix but consequently in a straight line in a projection on $X, S$ is a vector product of the projection of momentum and the radius vector of this particle on $Y$. In this case, the magnitude of momentum $S$ becomes $S=|\boldsymbol{S}|=\left|p_{y} a\right|=m c \hbar / m c=\hbar$. This formula retains some arbitrariness in the orientation of vector $S$ in six-dimensional space: it may be oriented in any direction in four-dimensional subspace perpendicular to the plane of rotation in $Y$. In the general case, vector $S$ has four non-zero components along directions perpendicular to each other and the plane of rotation of the particle in $Y$. In the case of rotation in the plane $y_{2}, y_{3}$, such components are $S_{1}, S_{2}, S_{3}, S_{4}$ along the axes $x_{1}, x_{2}$, $x_{3}, y_{1}$, respectively, and $S=\left(S_{1}^{2}+S_{2}^{2}+S_{3}^{2}+S_{4}^{2}\right)^{1 / 2}=\hbar$.

Components $S_{1}, S_{2}$, and $S_{3}$ are components of the spin of the particle, and $S_{4}$ is a projection of the isotopic spin of the particle. Thus, spin and isotopic spin are the projections on $X$ and $Y$, respectively. From (6), $p_{y}$ is independent of velocity $v$. Hence spin and isotopic spin are also independent of velocity $v$ and are not subject to the Lorentz transformation.

Vector $\boldsymbol{S}$, which remains perpendicular to the plane of rotation of the particle, has three degrees of freedom, and may be oriented in an arbitrary manner relative to those coordinate axes. A uniform distribution of components of the vector over the above four axes which are perpendicular to each other and the plane of rotation in $Y$ corresponds to particles with spin one-half. Then these components are equal to $+\hbar / 2$ or $-\hbar / 2$, and the sum of squares of these components in $X$ is equal to $(3 / 4) \hbar^{2}$.
In quantum mechanics, this is the "total" (in three-dimensional space) square of the proper momentum of a particle. Orientations of vector $\boldsymbol{S}$ obtained from previous orientations through allowable turns, keeping one or two given components invariable, are referred to the last case as well. So, if one of the components of the vector in $X$ and one component in $Y$ have a fixed value $+\hbar / 2$ or $-\hbar / 2$ then the vector retains the possibility of rotating about two corresponding axes. In this case, two nonfixed components will not have specific values (these are ordinary situations in quantum mechanics, where the absence of fixation of quantities is the exception rather than the rule). For equal allowed probabilities of orientations of that vector, the mean-square components mentioned above are equal to $\hbar / 2$. A change of direction of rotation of a particle about the axis of the motion tube in the opposite sense also changes the signs of the components to the opposite ones and corresponds to the transition to an antiparticle.

In the general case, the moment of momentum has four non-zero components along directions perpendicular each to other and a plane of rotation of a particle. Therefore, the theory of spin and isotopic spin must explicitly or implicitly use four coordinates and four projections of vectors on the axes of those coordinates. The total moment of momentum $\boldsymbol{M}$ in $R_{6}$ is the vector product of the total momentum $\boldsymbol{p}_{x}+m \boldsymbol{c}$ and radius vector $\boldsymbol{r}+\boldsymbol{a}$ of a particle in $R_{6}$, where $p_{x}$ and $\boldsymbol{r}$ denote the momentum and radius vector in $X$, and $m \boldsymbol{c}$ and $\boldsymbol{a}$ denote the momentum and radius vector in $Y$. Moment $\boldsymbol{M}$ is a four-dimensional vector perpendicular to the plane of rotation of a particle in $Y$. On average over a period of rotation about the axis of the tube, the cross-terms disappear and then $\boldsymbol{M}$ $=\boldsymbol{L}+\boldsymbol{S}$, where $\boldsymbol{L}$ is the orbital moment in $X$, and $s=\left[\begin{array}{ll}\boldsymbol{a} & \mathbf{m c}\end{array}\right]$ is the spin-isotopic spin moment of rotation in $Y$. Three components of $\boldsymbol{S}$ represent the spin projections $S_{1}, S_{2}$, and $S_{3}$ on $X$, and the component on $Y$ represents the isotopic spin $S_{4}$. Hence, on account of the mutual perpendicularity of vectors $\boldsymbol{a}$ and $\boldsymbol{c}$ and equalities $|\boldsymbol{a}|=a,|\boldsymbol{c}|=c$, one obtains $S=\hbar$. With the uniform distribution of components on four coordinate axes, which are perpendicular to the plane of rotation in $Y$, one finds $\left|S_{j}\right|=\hbar / 2, j=1,2,3,4, S_{1}^{2}+S_{2}^{2}+S_{3}^{2}=3 \hbar^{2} / 4$.

Of interest is the question of why the values of the proper momentum and its components in $X$ and $Y$, that is, spin and isotopic spin, are independent of the mass of an elementary particle. In six-dimensional treatment, the answer is obvious: the momentum is proportional to this mass but the radius of the Compton orbit in $Y$ for this particle is inversely proportional to this mass, and therefore the product of the momentum and radius of the Compton orbit is independent of this mass.

The proper magnetic moment $\boldsymbol{\mu}$ of a charged elementary particle is defined similarly to the proper mo- 
ment of momentum $S$ accordingly to the known formula of electrodynamics [10]:

$$
\boldsymbol{\mu}=\frac{e}{2 c}[\boldsymbol{R} c]
$$

where $\boldsymbol{R}$ is the six-dimensional radius vector of the particle, and $c$ is the vector of its velocity in $Y$. Since a contribution to this vector product gives only the projection $a$ of the radius vector $\boldsymbol{R}$ on subspace $Y$, one finds $\boldsymbol{\mu}=\frac{e}{2 c}[\boldsymbol{a} c]$. From this, accounting for the mutual perpendicularity of vectors $\boldsymbol{a}$ and $\boldsymbol{c}$ as well as equalities $|\boldsymbol{a}|=a$ and $|\boldsymbol{c}|=c$, one finds the magnitude $\mu$ of the proper moment $\boldsymbol{\mu}$ of the particle which is equal to the Bohr magneton:

$$
\mu=|e| a / 2=\frac{\hbar}{2 m c}=\mu_{B} .
$$

In the simplest case, when the vector $\boldsymbol{\mu}$ has no components in subspace $Y$, the components of this vector define in $X$ a three-dimensional vector whose magnitude is equal to the Bohr magneton.

A projection $\mu_{x}$ of the magnetic moment onto an arbitrary chosen direction (called the axis of quantization) in subspace $X$ may have a fixed value only in the case when the projection of the proper moment of momentum has a fixed value as well. In this case, according to (11), $\mu_{x}= \pm \mu_{B}$. With a uniform distribution of components of the proper moment of momentum over four axes which are perpendicular each to other and a plane of rotation of a particle in $Y$, in the case considered $S_{x}= \pm m c a / 2$, which equals $+1 / 2$ or $-1 / 2$ (in units of $\hbar$ ). Therefore $\mu_{x} / S_{x}=e / m c$, in accordance with the experiment of Stern and Gerlach.

A particle which is at rest in $X$ travels in the circle of radius $a_{\infty}$ at the speed of light $c$. The above centripetal cosmological force in $Y$ corresponding to this circular motion is $F_{0}=p_{y} c / a_{\infty}=m c^{2} / a_{\infty}, c^{2} / a g$, times larger than the weight of the particle at the Earth's surface. It is $2.38 \times 10^{28}$ for an electron. The same result is obtained when the particle moves along the helix:

$F_{0}=p c K / \cos \theta$, where $K=\cos ^{2} \theta / a_{\infty}$ is the curvature of the helix. Here it is evident that $F_{0}=m c^{2} / a_{\infty}$ at any $\theta$.

Let us show that the force of electrical interaction between two electrons in six-dimensional space is $e^{2} / R^{2}$, the generalized Coulomb force, if the Biot-Savart formula holds true for this space, where $R$ is the distance between charges $e$ in the complete space.

The disposition of two electrons on the opposite sides of the same tube of motion has an energetic advantage, whereby the distance between them in the complete space is equal to $R=\sqrt{r^{2}+4 a^{2}}$, where $r$ is the distance between projections of the particles onto $X$, and $a$ is the distance of them from the axis of their rotation in $Y$. The tube radius $a$ here depends on $r$ and tends asymptotically to $a_{\infty}=\hbar /(m c)$ with increases in $r$, with $m$ and $c$ being the mass of a particle and the speed of light at infinity, respectively. Due to such a rotation with the shift in phase $\pi$ between two particles, the Coulomb force of their repulsion in the complete space is equal to $e^{2} / R^{2}$, where $e$ is the charge of the electron. Projections of this force onto subspaces $X$ and $Y$ are $F_{\|}=\left(e^{2} / R^{2}\right) \sin \chi$ and $F_{\perp}=\left(e^{2} / R^{2}\right) \cos \chi$, respectively, where $\sin \chi=r / R$ and $\cos \chi=2 a / R$, so that $F_{\|}=e^{2} r / R^{3}$, $F_{\perp}=2 e^{2} a / R^{3}$, and $\sqrt{F_{\|}^{2}+F_{\perp}^{2}}=e^{2} / R^{2}$. The force $F_{\perp}$ reacts against the centripetal force $F_{0}=m c^{2} / a_{\infty}$. Therefore, the radius of rotation $a$ is a little in excess of the tube radius $a_{\infty}$ at infinity.

Applying the Biot-Savart formula to six-dimensional space, the total magnetic field of the charge at rest in $X$ is defined at the distance $R$ from the charge $e$ as

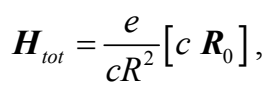

where $\boldsymbol{R}_{\mathbf{0}}$ is the unit vector directed from the charge to the point of observation, and $c$ is the velocity of the charge. When $R$ is the distance between two electrons,

$$
\begin{aligned}
\boldsymbol{R}_{0} & =\boldsymbol{r}_{0} \sin \chi+\boldsymbol{a}_{0} \cos \chi \\
& =\boldsymbol{r}_{0}(r / R)+\boldsymbol{a}_{0}(2 a / R), \\
\boldsymbol{H}_{\text {tot }} & =\frac{e}{c R^{2}}\left[\begin{array}{cc}
\boldsymbol{c}_{0} & \boldsymbol{R}_{0}
\end{array}\right] \\
& =\frac{e}{R^{2}}\left\{\left[\begin{array}{ll}
\boldsymbol{c}_{0} & \boldsymbol{r}_{0}
\end{array}\right](r / R)+\left[\boldsymbol{c}_{0} \boldsymbol{a}_{0}\right](2 a / R)\right\}
\end{aligned}
$$

where $\boldsymbol{r}_{0}$ is a unit vector along the radius vector $\boldsymbol{r}$ in $X$, $\boldsymbol{a}_{0}$ is the unit vector along the radius vector of the charge $e$ in the plane of rotation in $Y$, and $c_{0}$ is the unit vector along velocity $c$. Let us show that the Coulomb force of interaction between the two charges $\left(e\right.$ and $\left.e^{\prime}\right)$ is the Lorentz force acting on these charges moving in $Y$. Referring to (12) this force is equal to

$$
\begin{aligned}
& \boldsymbol{f}=\frac{e^{\prime}}{c}\left[\begin{array}{ll}
c^{\prime} & H_{t o t}
\end{array}\right] \\
& =\frac{e^{\prime} e}{c R^{2}}\left\{\left[c^{\prime}\left[\begin{array}{ll}
\boldsymbol{c}_{0} & \boldsymbol{r}_{0}
\end{array}\right]\right](r / R)+\left[\boldsymbol{c}^{\prime}\left[\boldsymbol{c}_{0} \boldsymbol{a}_{0}\right]\right](2 a / R)\right\}
\end{aligned}
$$

From this, and taking into account that for two interacting electrons $c^{\prime}=-c$,

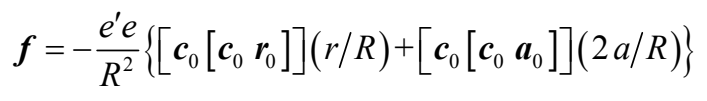

Revealing the triple vector products and taking into account the mutual perpendicularity of the vectors involved and the fact that in the case under consideration $c^{\prime}=-c$, one obtains 


$$
\begin{aligned}
& {\left[c_{0}\left[c_{0} r_{0}\right]\right]=-r_{0},\left[c_{0}\left[c_{0} a_{0}\right]\right]=-a_{0},} \\
& f=\frac{e^{\prime} e}{R^{3}} r r_{0}+\frac{e^{\prime} e}{R^{3}} 2 a a_{0} .
\end{aligned}
$$

In the last formula the first term represents the projection of the generalized Coulomb force onto $X$, and the second term is its projection onto $Y$. Their magnitudes are equal to $F_{\|}$and $F_{\perp}$, respectively. From this it is seen that the electric forces (of the Lorentz type as well) are due to the movement of charges in subspace $Y$ in the magnetic field arising because they are orbiting in $Y$, in contrast to the usual magnetic forces which are caused by the movement of charges in the same subspace $X$. The force $F_{\|}$is equal to zero at $r=0$. This is the point of indifferent equilibrium, near which electrons may be comparatively slow moving for a long time if they are ejected for head-on collision one to other with the appropriate original kinetic energy [7].

When there is a change of the course of time, the direction of rotation of particles in $Y$ is reversed, which causes the signs of the fields to change to the opposite ones. In this way the corresponding trajectories in the complete space occur as though they were reflected from a mirror. The motion of a particle along the helix (of the Compton radius in $Y$ ) with a revolution to the left (right), viewed in the direction of travel, is changed into motion along the mirror-reflected helix with rotation to the right (left). The sign of the charge may be regarded as nothing but a mark corresponding to one or the other (positive or negative) direction of rotation of a particle in the space of extra dimensions. In contrast to the standard formulation of the CPT theorem, in which the properties of particles and antiparticles, respectively, under the direct and reverse courses of time are juxtaposed, in the six-dimensional treatment of CPT symmetry the properties of the same elementary particle are juxtaposed under the direct and the reverse course of time. In this treatment the charges of particles and antiparticles are the same but the signs of the corresponding electrical and magnetic fields are defined by the direction of rotation in the extra-dimensional space. The corresponding formulation of the theorem is as follows. If the course of time is reversed, the particle moves in the complete space backward along the same trajectory of this particle as under the direct course of time. In this way the signs of the fields automatically change to the opposite ones, and the trajectory, viewed in the direction of travel, in the complete space occurs as though it were reflected in a mirror, so that this particle acquires all of the properties of the antiparticle [7].

Inertia and the pressure of light are phenomena of the same nature because all elementary particles move in the complete space at the speed of light. Both are due to changes of orientation of the momentum of elementary particles in the complete space under the action of an external force.

The six-dimensional treatment of gravitation which corresponds to the motion of particles with the speed of light in the Compton neighbourhood of the three-dimensional space along the geodesics complying with the Fermat principle leads to the Papapetrou metric and gravitational waves [11-13]. The envelope of the geodesics has the form of a tubular surface with the Compton transverse sizes in the additional subspace where the radius and speed of light vary along the tube. The force of gravity is the projection of cosmological force $F_{0}$ on the meridian of that tubular surface. Gravitational waves, which are perturbations of these radii and speed, turn out to attenuate exponentially here. Their amplitudes are considered in the near-field zone of the rotator with $n$ Maltese cross lobes and are calculated at $n=4$ [12].

\section{REFERENCES}

[1] F. Klein, "Uber Neuere Englische Arbeiten zur Gesammelte Matematishe Abhandlungen," Springer, Berlin, 1922.

[2] F. Klein, "Vorlezungen über die höhere Geometrie," Ohne Auftrag in Berlin, Vol. 3, 1926, p. 219.

[3] A. A. Margolin, "Principle of Simplicity," Chemistry and Life, Vol. 9, 1981, p. 79.

[4] Yu. B. Rumer, "Investigations on 5-Optics," Gostekhizdat, Moscow, 1956.

[5] I. A. Urusovskii, "Six-Dimensional Treatment of the Relativistic Mechanics and Spin, Metric Gravitational Theory, and the Expanding Universe," Uspekhi Sovremennoi Radioelectroniki. Zarubezhnaya Radioelectronika, Vol. 3, 1996, p. 3.

[6] I. A. Urusovskii, "Six-Dimensional Treatment of the Quark Model of Nucleons," Uspekhi Sovremennoi Radioelectroniki. Zarubezhnaya Radioelectronika, Vol. 6, 1999, pp. 64-74.

[7] I. A. Urusovskii, "Six-Dimensional Treatment of CPTSymmetry," Proceedings of International Scientific Meeting Physical Interpretations of Relativity Theory, Moscow, 4-7 July 2005, pp. 318-326.

[8] R. O. di Bartini, "Several Relations between Physical Constants," Doklady Akademii Nauk SSSR, Vol. 163, No. 4, 1965, pp. 861-864.

[9] I. A. Gribov, "Dark Matter as Pico-Windows to Physically Equal Multiverse," 2012.

http://www2.hu-berlin.de./leibniz-sozietaet/journal/archiv e/13_12/01_gribov.pdf

[10] J. D. Jackson, "Classical Electrodynamics," Wiley, New York \& London, 1962.

[11] I. A. Urusovskii, "Gravity as a Projection of the Cosmological Force," Proceedings of International Scientific Meeting Physical Interpretations of Relativity Theory, Moscow, 30 June-3 July 2005, pp. 359-367.

[12] I. A. Urusovskii, "Gravitational Waves and Papapetrou 
Metric in the Six-Dimensional Treatment of Gravitation," Physics of Wave Phenomena, Vol. 18, No. 3, 2010, pp. 185-195. doi:10.3103/S1541308X10030064
[13] A. Papapetrou, "Eine Theorie des Gravitationsfeldes mit einer Feldfunrtion," Zeitschrift fur Physik, Vol. 139, 1954, pp. 518-532. doi:10.1007/BF01374560 